\title{
Prevalence Rate and Functional Status of Cerebellar Ataxia in Korea
}

\author{
Byung-Euk Joo $\cdot$ Chan-Nyoung Lee $\cdot$ Kun-Woo Park
}

Published online: 6 December 2011

(C) The Author(s) 2011. This article is published with open access at Springerlink.com

\begin{abstract}
Cerebellar ataxia (hereinafter referred to as CA) designate a group of neurodegenerative disorders. $\mathrm{CA}$ is distinguished into a group of hereditary and nonhereditary disorders. CA shows clinically progressive features and accompanies various neurological abnormalities. However, there are very few studies and case reports in Korean patients. To estimate the prevalence rate and current status of the CA patients in Korea, we used data from the Health Insurance Review and Assessment Service (HIRAS) and from the National Health Insurance Corporation. To evaluate the functional status of CA patient in Korea, we conducted a simple random sampling among the 500 members of Korea Ataxia Society registered on its homepage. We evaluated the functional status and degree of disturbance to their everyday life with modified Rankin scales and Barthel ADL index. Using the data from HIRAS, we could estimate the prevalence rate of CA patients in Korea as 8.29 patients $/ 100,000$ persons. The prevalence rate of hereditary and non-hereditary cerebellar ataxia was 4.99 patients/100,000 persons and 3.30 patients/ 100,000 persons, respectively. Data on rare intractable diseases reported by the Korean Centers for Disease Control and Prevention in 2006 suggested that the number of CA patients who have visited medical institutes was almost doubled for the 2-year period.
\end{abstract}

B.-E. Joo $\cdot$ C.-N. Lee $\cdot$ K.-W. Park

Department of Neurology, Korea University Medical Center,

Seoul, South Korea

K.-W. Park $(\square)$

Korea University College of Medicine,

\#126-1, Anam-Dong 5Ga, Seongbuk-Gu,

Seoul 136-705, South Korea

e-mail: kunu@korea.ac.kr
The medical expense and hospital stay also increased 4.5- and 3-fold, respectively. After severity evaluation with modified Rankin scales and Barthel ADL index, we found that most CA patients in Korea have ataxiarelated difficulties in their everyday life.

Keywords Cerebellar ataxia $\cdot$ Prevalence rate $\cdot$ Functional status

\section{Introduction}

Cerebellar ataxia (hereinafter referred to as CA) designate a group of neurodegenerative disorders. CA is distinguished into a group of hereditary and non-hereditary disorders [1, 2]. Hereditary CA can have an autosomal-dominant, autosomal-recessive, X-linked, or mitochondrial mode of inheritance [1]. Non-hereditary CA can be symptomatic or idiopathic, and symptomatic CA may be caused by multiple causes including malformations, toxic agents, endocrine disorders, and infections. CA shows clinically progressive features and accompanies various neurological abnormalities. The symptoms include not only walking difficulty due to cerebellum degeneration, but also optic atrophy, ophthalmoplegia, retinitis pigmentosa, sensory loss, and dysarthria $[3,4]$. These symptoms cause many difficulties in the everyday life of CA patients [2]. Although the patients have a lot of difficulties with their everyday life, CA is such a rare disease that there have been only few studies and case reports in Korea [5]. This study aimed to provide useful data for establishing a more appropriate national policy by identifying the prevalence rate of $\mathrm{CA}$ and examining its current status in collaboration with Korea Centers for Disease Control and Prevention, conclusively to increase the social awareness of CA. 


\section{Methods}

\section{Sources of Patient Data}

There are two main organizations providing national health insurance in Korea: the National Health Insurance Corporation (NHIC) and the Health Insurance Review and Assessment Service (HIRAS). NHIC is the organization which provides health care benefits in cases of illness and injuries, to enhance public health and strengthen social security. Data from NHIC were derived from the payment request sheet generated from each visit to hospitals and both inpatient or outpatient clinics. NHIC covers most Korean citizens and registered residents of foreign nationality, except special population groups, such as foreign military personnel. It encompasses all hospital visits nationwide. HIRAS is the organization which reviews whether the medical service fee was fairly charged in accordance with the law, to maintain the quality standard, as well as an adequate level of medical fees. Data from HIRAS also covers all hospital visits nationwide.

\section{Prevalence Rate Survey}

To estimate the prevalence rate of CA patients in Korea, our research team used data from the HIRAS. First, we selected the patients who were candidates for the health insurance and medical care review and assessment, from 1 January 2008 to the end of December 2008, using data from HIRAS. Then, we sampled those whose principal and secondary diagnosis codes were registered as G11 in the International Classification of Disease-10 (hereinafter ICD10; Table 1). Ataxia is not a main symptom in hereditary spastic paraplegia (G11.4); therefore, those patients were excluded from our database. Then patients were classified across year, and overlapping data was also removed, based on the serial number. In cases when the major and minor diagnosis codes differed, the major diagnosis code was set as the main criterion. Thus, the patients were reclassified according to the sub-diagnosis codes within G11 and we established a database of presumed CA patients in Korea, according to the G11 diagnosis code and based on information gathered by HIRAS between 2006 and 2008 . We classified the patients registered as G11.0, G11.1, G11.3, G11.8, and G11.9 as hereditary CA and those diagnosed with G11.2 as non-hereditary cerebellar ataxia, and estimated the prevalence rate in each group, as presented in Fig. 1.

\section{Health Care Status Survey in CA Patients in Korea}

To examine the current health care status of the CA patients in Korea, we used data from the NHIC. We sampled the patients, whose diagnosis codes corresponded to G11 of ICD-10 from the data registered at NHIC as of December, 2008. Then, the patients registered as G11.4 were excluded from estimating the prevalence rate, as described above.

For each group, we examined the annual medical expense per capita, hospital stay per capita, distribution of medical care, and registration as a disabled person.

\section{The Functional Status Evaluation of CA Patients}

In this study, we originally designed the functional status evaluation of the CA patients as follows: First, we planned a simple random sampling of the database and follow-up study using the medical records. We also planned telephone interview or visiting survey to evaluate the severity in cases when the medical records were insufficient or unavailable. However, it was impossible to identify individual patients through the data from HIRAS due to the "Law of Private Information Protection in Public Institutions". Therefore, to evaluate the severity, we conducted a simple random sampling among the 500 members of Korea Ataxia Society registered on its homepage. Following this process, we conducted telephone interviews or home visits to administer the modified Rankin scales and Barthel ADL index in order to evaluate the severity of $\mathrm{CA}$ and the degree of disturbance to the patients' everyday life.

Table 1 Comparison on the using rate of medical service in cerebellar ataxia patients

\begin{tabular}{lllll}
\hline & & Number & Medical expense: Korean won (US dollar: USD) & Hospital visiting days \\
\hline 2008 & Hereditary & 1,243 & $1,181,139$ won (about 984.3USD) & 13.9 \\
& Non-hereditary & 943 & $1,544,979$ won (about 1,287.5 USD) & 17.7 \\
\multirow{2}{*}{2006} & Total & 2,186 & $2,726,118$ won (about 2,271.8 USD) & 31.6 \\
& Total & 1,450 & 595,189 won (about 496.0 USD) & 10.2 \\
\hline
\end{tabular}

Hereditary cerebellar ataxia with hereditary, Non-hereditary cerebellar ataxia without hereditary, Medical expense (Korean won), medical expense per capital, Hospital visiting days hospital visiting days per capital 


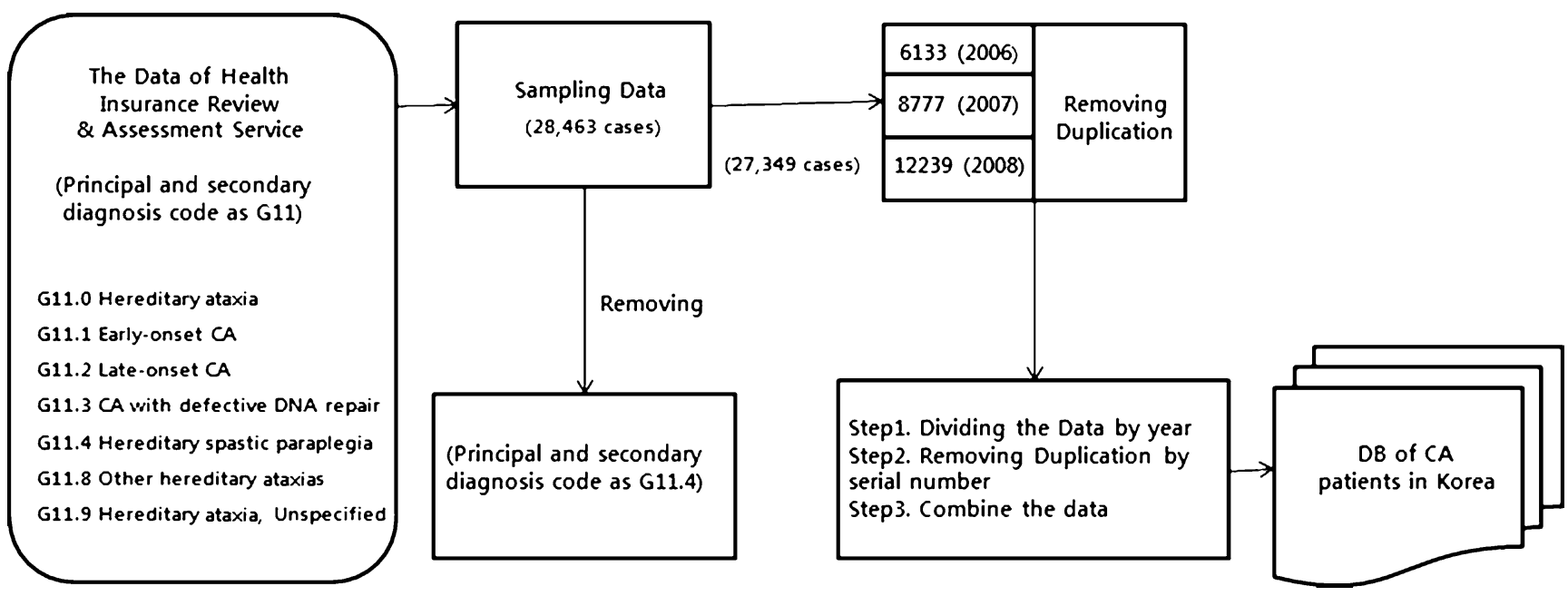

Fig. 1 The flowchart about building of the database with the data from Health Insurance Review and Assessment Service. $C A$ cerebellar ataxia)

\section{Results}

Prevalence of CA in Korea

According to data from HIRAS, the number of patients estimated to have CA in Korea was 3,996. To estimate the prevalence rate of CA, we used the entire number of people who applied to HIRAS in 2008 (i.e., 48,606,000 persons) as the study population and estimated the prevalence rate of CA patients in Korea as 8.22 patients/100,000 persons.

When the 3,996 CA patients were classified according to the sub-diagnosis code G11, the non-hereditary CA patients were 1,594 (39.9\% of the entire CA patients) and hereditary CA patients were 2,402 (60.1\%). Thus, we estimated the prevalence rate of hereditary CA patients as 4.94 patients/ 100,000 persons and non-hereditary CA patients as 3.27 patients/100,000 persons, respectively.
With respect to the gender ratio for the hereditary and the non-hereditary CA groups, there were 1,279 men $(53.2 \%)$ and 1,123 women $(46.8 \%)$ in the hereditary CA group, whereas there were 908 men $(57.0 \%)$ and 686 women $(43.0 \%)$ in the non-hereditary group. These findings show that male patients are more frequently observed than women in both groups.

With respect to the age-specific prevalence rate, as presented in Fig. 1, there were 226 patients of age 09 years, 149 of age 11-19 years, 202 of age 20-29 years, 272 of age $30-39$ years in the hereditary CA group, which suggested that $849(35.3 \%)$ out of total 2,402 were diagnosed under the age of 40 . In contrast, non-hereditary CA was mainly diagnosed over the age of 30, with 463 patients of age 50-59 years, 534 of age 60-69 years, and 363 of age 70 years and above. Of the 1,549 patients, 1,360 $(85.3 \%)$ were aged 50 or above, as presented in Fig. 2.
Fig. 2 Comparison of numbers according to age between CA patients with and without hereditary. Hereditary cerebellar ataxia with hereditary,

Non-hereditary cerebellar ataxia without hereditary

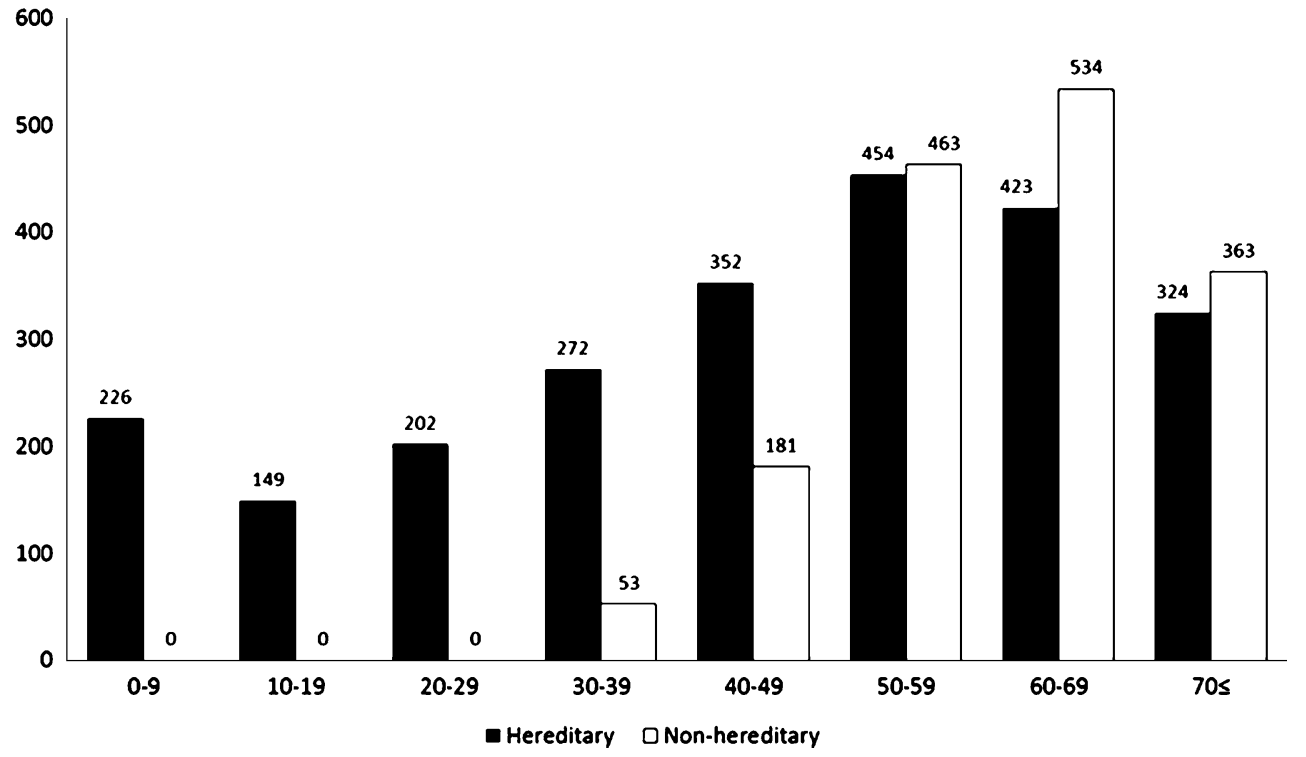




\section{Health Care Status Survey in CA Patients in Korea}

We were able to identify the number of CA patients, the annual medical expense per capita and the annual hospital stay per capita, as of December 2008. Comparing these results to the reports on rare intractable diseases published by the Korea Center for Disease Control and Prevention in 2006, it showed that the number of CA patients who have visited medical institutes was almost doubled for the 2-year period. The medical expense per patient increased 4.5-fold, from 595,189 Korean won (about 496.00 U.S. dollars, USD) to 2,726,118 Korean won (about 2,271.8 USD), and the hospital stay also increased 3.1-fold, as shown in Table 1.

\section{The Functional Status Evaluation of CA Patients}

To evaluate the severity of CA, we randomly sampled the 500 members of Korea Ataxia Society and assigned them to two study groups, i.e., hereditary and non-hereditary CA, with 34 sample size for each group. With respect to the gender ratio for each group, there were 19 men and 15 women in each group. The age distribution for the hereditary CA group is presented in Table 2 .

All patients were surveyed with the modified Rankin scale via telephone interviews or home visits to evaluate the severity of CA.

Our results showed that $55.9 \%$ of the hereditary CA patients reported grade 0,1 , or 2 symptoms, but no major disabilities, so they were able to achieve ordinary self-care. There were $11.7 \%$ with grade 3 who had moderate disabilities and needed some assistance, but could walk without assistance; $14.7 \%$ with grades 4 and 5 had severe disabilities and were in bedridden state.

In contrast, there were $64.7 \%$ of non-hereditary CA patients with grade 4 or 5 symptoms who had severe disabilities, could not walk without assistance, and required assistance for all basic physical needs. These results suggest

Table 2 Distribution of age in a sample was selected from the 500 cerebellar ataxia patients in Korea Ataxia Society through the random sampling method

\begin{tabular}{lllll}
\hline & Hereditary & Ratio (\%) & Non-hereditary & Ratio (\%) \\
\hline$N$ & 34 & & 34 & \\
M/F & $19: 15$ & & $19: 15$ & \\
Age & & & & \\
$<20$ & 4 & 12 & 0 & 0 \\
$30-39$ & 9 & 26 & 3 & 9 \\
$40-49$ & 12 & 35 & 2 & 85 \\
$50 \leq$ & 9 & 26 & 29 & 6 \\
\hline
\end{tabular}

Hereditary cerebellar ataxia with hereditary, Non-hereditary cerebellar ataxia without hereditary
Table 3 The functional status evaluation with Modified Rankins scales in Cerebellar ataxia patients

\begin{tabular}{lcc}
\hline Grade & Hereditary (\%) & Non-hereditary (\%) \\
\hline Mild $(0,1,2)$ & $19(55.9 \%)$ & $10(29.4 \%)$ \\
Moderate $(3)$ & $4(11.8 \%)$ & $2(5.9 \%)$ \\
Severe $(4,5)$ & $11(32.3 \%)$ & $22(64.7 \%)$ \\
Total & $34(100 \%)$ & $34(100 \%)$ \\
\hline
\end{tabular}

Hereditary cerebellar ataxia with hereditary, Non-hereditary cerebellar ataxia without hereditary

$P$ value 0.012

that more than $60 \%$ of non-hereditary CA patients show severe or above level of disabilities (Table 3).

To assess the ability to perform everyday life activities in each group, the Barthel ADL index was administered via telephone interview or home visits. The results showed that $58.5 \%$ of hereditary CA patients scored $17-20$ on the Barthel ADL index and had only minor difficulties with everyday life activities; only $17.6 \%$ had severe difficulties and scored 4 or below.

In contrast, in the non-hereditary CA group, $29.4 \%$ had minor difficulties with everyday life activities (score 1720 ), and up to $49.9 \%$ showed severe or above level of disabilities, as presented in Table 4.

\section{Discussion}

In Korea, there is a lack of objective studies on the prevalence rate of CA patients, current status of medical service use, registration as a disabled person, and severity evaluation. This was an extensive and objective study with significant results, using national data from both HIRAS and NHIC. We established our database according to the diagnosis code registered in the national statistical data. Thus, ataxia related to diseases other than CA, such as Parkinson's disease, hydrocephalus, myelopathy, and peripheral nerve disease, could be included, if the symptoms were registered as ataxia-related diagnosis code in the

Table 4 The functional status evaluation with Barthel index in Cerebellar ataxia patients

\begin{tabular}{lcc}
\hline Grade & Hereditary (\%) & Non-hereditary (\%) \\
\hline $0-4$ (severe) & $6(17.6 \%)$ & $10(29.4 \%)$ \\
$5-16$ (moderate) & $8(23.6 \%)$ & $14(41.2 \%)$ \\
$17-20$ (mild) & $20(58.8 \%)$ & $10(29.4 \%)$ \\
Total & $34(100 \%)$ & $34(100 \%)$ \\
\hline
\end{tabular}

Hereditary cerebellar ataxia with hereditary, Non-hereditary cerebellar ataxia without hereditary

$P$ value 0.034 
medical record. Conversely, current CA patients could be excluded if the ataxia-related diagnosis code was not entered. Therefore, we cannot rule out the possibility that some of the data used in this study may be under/ overestimated or less accurate.

Studies about the prevalence rate in all CA patients, including hereditary and non-hereditary $\mathrm{CA}$, are very rare. Several nationwide surveys have been performed in Japan [6-7]. In 2008, the prevalence rate of CA in Japan was $18.5 / 100,000$ [6]. Similarly, a study about the prevalence rate of CA was conducted in the Hokuriku district in Japan, from 2005 to 2006, and CA prevalence in that district was $31.1 / 100,000$ [7]. In our study, the overall prevalence rate of CA in Korea was 8.29 per 100,000 , which was lower compared to results in Japan. This difference might be attributable to regional and study method differences.

Studies about the prevalence rate and the dominant subtype of hereditary CA have been conducted in many countries [8-12]. In many studies, the prevalence rate of autosomal dominant cerebellar ataxia (ADCA) was 1-4/ 100,000 [8-12]. In our study, hereditary CA compromised $60.1 \%$ of all CA cases, and the proportion suggested that the prevalence rate of hereditary CA patients was about $4.98 / 100,000$, which is a little higher than those reported in other West and Asian countries, but lower than the prevalence of hereditary CA in Japan.

Several Asian countries also reported the dominant subtype of ADCA [1, 13-16]. Spinocerebellar ataxia type 3 (SCA3) was the commonest subtype worldwide [17]. The frequency of SCA3 was higher than that of other subtypes of ADCA in China, Singapore, and Japan [6, 13-15]. Several frequency studies according to ADCA subtype were conducted in Korea [18-21]. In one study, the most frequent subtype was SCA3, followed by SCA2 [21]. However, most studies reported that SCA2 was the most frequent ADCA [18-20]. Those studies showed that in Korea, the subtype frequencies of SCA are different from those in neighboring countries.

The survey on the current status of medical service used by CA patients showed that the number of patients increased by as much as $50 \%$, compared to the prevalence rate survey report of rare and intractable diseases in 2006, and the medical expense and hospital stay per patient increased 4.5- and 3-fold, respectively.

The severity evaluation study through random sampling was conducted with a specific sample, which may not be representative for the entire population. In addition, the possibility of selection error in constructing the sample cannot be ruled out. However, considering the very low CA prevalence rate, it can be assumed that the severity evaluation results with the random sample may have significant implications.

In the severity evaluation study, we found $55.9 \%$ hereditary CA patients who scored gradse 0,1 , and 2 on the modified Rankin scale, whereas those who scored 17 to 20 on the Barthel index were $58.8 \%$. These results showed that more hereditary CA patients had minor disabilities. In contrast, in the non-hereditary CA group, there were $64.7 \%$ patients with grade 4 or below who were not able to walk without assistance and $64.7 \%$ had severe or above level of disability scoring less than 12 on the Barthel Index. These results may be attributed to the fact that the number of older patients was increased in the non-hereditary CA, compared to hereditary CA, which in turn may lead to more severe disability level.

\section{Conclusion}

This study is the first report on the prevalence rate and current status of CA in Korea using national statistical data. It is expected that the social awareness of CA patients would increase, leading to improvement in the relevant systems in the future.

Acknowledgment This study was granted by the Korean Center for Disease Control and Prevention. (R0903001, KW PARK)

Conflict of Interests There is no conflict of interest in the work presented in this manuscript.

Open Access This article is distributed under the terms of the Creative Commons Attribution Noncommercial License which permits any noncommercial use, distribution, and reproduction in any medium, provided the original author(s) and source are credited.

\section{References}

1. Brusse E, Maat-Kievit JA, van Swieten JC. Diagnosis and management of early- and late-onset cerebellar ataxia. Clin Genet. 2007;71:12-24.

2. Manto MU. The wide spectrum of spinocerebellar ataxias (SCAs). Cerebellum. 2005;4:2-6.

3. Evidente VG, Gwinn-Hardy KA, Caviness JN, Gilman S. Hereditary ataxias. Mayo Clin Proc. 2000;75:475-90.

4. Manto M, Marmolino D. Cerebellar ataxias. Curr Opin Neurol. 2009;22:419-29.

5. Bang OY, Huh K, Lee PH, Kim HJ. Clinical and neuroradiological features of patients with spinocerebellar ataxias from Korean kindreds. Arch Neurol. 2003;60:1566-74.

6. Tsuji S, Onodera O, Goto J, Nishizawa M. Sporadic ataxias in Japan - a population-based epidemiological study. Cerebellum. 2008;7:189-97.

7. Shibata-Hamaguchi A, Ishida C, Iwasa K, Yamada M. Prevalence of spinocerebellar degenerations in the Hokuriku district in Japan. Neuroepidemiology. 2009;32:176-83.

8. Leone M, Bottacchi E, D'Alessandro G, Kustermann S. Hereditary ataxias and paraplegias in Valle d'Aosta, Italy: a study of prevalence and disability. Acta Neurol Scand. 1995;91:183-7. 
9. van de Warrenburg BP, Sinke RJ, Verschuuren-Bemelmans CC, Scheffer H, Brunt ER, Ippel PF, et al. Spinocerebellar ataxias in the Netherlands: prevalence and age at onset variance analysis. Neurology. 2002;58:702-8.

10. Infante J, Combarros O, Volpini V, Corral J, Llorca J, Berciano J. Autosomal dominant cerebellar ataxias in Spain: molecular and clinical correlations, prevalence estimation and survival analysis. Acta Neurol Scand. 2005;111:391-9.

11. Silva MC, Coutinho P, Pinheiro CD, Neves JM, Serrano P. Hereditary ataxias and spastic paraplegias: methodological aspects of a prevalence study in Portugal. J Clin Epidemiol. 1997;50:137784.

12. Koht J, Tallaksen CM. Cerebellar ataxia in the eastern and southern parts of Norway. Acta Neurol Scand Suppl. 2007; 187:76-9.

13. Tang B, Liu C, Shen L, Dai H, Pan Q, Jing L, et al. Frequency of SCA1, SCA2, SCA3/MJD, SCA6, SCA7, and DRPLA CAG trinucleotide repeat expansion in patients with hereditary spinocerebellar ataxia from Chinese kindreds. Arch Neurol. 2000;57:540-4.

14. Jiang H, Tang BS, Xu B, Zhao GH, Shen L, Tang JG, et al. Frequency analysis of autosomal dominant spinocerebellar ataxias in mainland Chinese patients and clinical and molecular characterization of spinocerebellar ataxia type 6. Chin Med J (Engl). 2005;118:837-43.
15. Zhao Y, Tan EK, Law HY, Yoon CS, Wong MC, Ng I. Prevalence and ethnic differences of autosomal-dominant cerebellar ataxia in Singapore. Clin Genet. 2002;62:478-81.

16. Dragasević NT, Culjković B, Klein C, Ristić A, Keckarević M, Topisirović I, et al. Frequency analysis and clinical characterization of different types of spinocerebellar ataxia in Serbian patients. Mov Disord. 2006;21:187-91.

17. Schols L, Bauer P, Schmidt T, Schulte T, Riess O. Autosomal dominant cerebellar ataxias: clinical features, genetics, and pathogenesis. Lancet Neurol. 2004;3:291-304.

18. Jin DK, Oh MR, Song SM, Koh SW, Lee M, Kim GM, et al. Frequency of spinocerebellar ataxia types 1, 2, 3, 6, 7 and dentatorubral pallidoluysian atrophy mutations in Korean patients with spinocerebellar ataxia. J Neurol. 1999;246:207-10.

19. Lee WY, Jin DK, Oh MR, Lee JE, Song SM, Lee EA, et al. Frequency analysis and clinical characterization of spinocerebellar ataxia types 1, 2, 3, 6, and 7 in Korean patients. Arch Neurol. 2003;60:858-63.

20. Kim HJ, Jeon BS, Lee WY, Chung SJ, Yong SW, Kang JH, et al. SCA in Korea and its regional distribution: a multicenter analysis. Parkinsonism Relat Disord. 2011;17:72-5.

21. Kim JY, Park SS, Joo SI, Kim JM, Jeon BS. Molecular analysis of Spinocerebellar ataxias in Koreans: frequencies and reference ranges of SCA1, SCA2, SCA3, SCA6, and SCA7. Mol Cells. 2001;12:336-41. 